\title{
Opinion Article Influence of Androgen Levels on Conception Probability in Patients Undergoing Fertility Treatment
}

\author{
Sebastian Findeklee* \\ Department for Gynecology, Saarland University Hospital, Germany
}

*Corresponding author: Sebastian Findeklee, Department for Gynecology, Saarland

Received Date: May 11, 2019

University Hospital, Obstetrics and Reproductive Medicine, Germany.

\section{Opinion}

Sterility is becoming an increasing issue, with estimated every seventh couple experiencing an unfulfilled desire to have children for more than a year [1]. As fulfilling the wish for a child is postponed in later periods of time by more and more women there is an increasing number of reproductive treatments, especially in-vitro fertilization (IVF) and intracytoplasmatic sperm injection (ICSI). Up to now, more than eight million children have been born after IVF or ICSI worldwide [2]. There are several potential causes for sterility. The three main reasons for sterility are disorders of ovarian function, anatomical causes, as pathological tubal patency or diminished motility of the tubes, and andrological subfertility [3]. Around $30 \%$ of all infertile women have anovulatory cycles. Mostly, this is attributed to polycystic ovaries (PCOS) characterized by high androgen levels, oligo- amenorrhea and enlarged ovaries $>$ $34 \mathrm{~mm}$ with at least 20 peripheral antral follicles $<10 \mathrm{~mm}$ [4]. The relationship between hyperandrogenaemia and sterility has already been clearly proven. At the same time an increasing evidence for low androgen levels being a risk factor for sterility in women can be detected within the literature. Nevertheless, data regarding the connection between female androgen levels and sterility, as well as conception rates after fertility treatment in these patients are sparse and rather contradictive [5].

At the beginning of this year our research group did publish the results of a monocentre retrospective study showing that there does not exist a significant correlation between female androgen blood concentrations and reproductive outcomes. However, there was a trend to higher conception rates after fertility treatment in women with higher blood concentrations of testosterone, androstendione and dehydroepiandrosteronsulfat [6]. We conclude that the relationship between serum androgen levels in women and sterility as well as reproductive outcomes should be investigated in larger prospective multicentre studies. Although hypoandrogenaemia is almost not considered with high awareness in women under sterility therapy it seems to be logical that it might be a risk factor for unsuccessful treatment. Androgens as well as estrogens count to steroid derivates being the main products of ovarian synthesis. A disturbed ovarian function goes along with a lower conception probability even under sterility therapy. Premature ovarian failure being regarded as the extreme form of ovarian dysfunction is characterized of cumulative pregnancy rates $<5 \%$ [7]. Based on these pathophysiologic findings several therapeutic approaches have been introduced into reproductive medicine within the last years. For improving conception rates a substitution with androgens, especially dehydroepiandrosterone (DHEA), is recommended by several working groups-particularly before the use of assisted reproductive technologies. Interestingly, for some years - even though experimental - androgens, mostly DHEA in a dose of $25 \mathrm{mg}$ three times a day, have been applied to women with limited ovary reserve over a period of 2-3 months before starting hormonal stimulation treatment. This should stimulate follicles to grow and the amount of punctured eggs to increase. Triantafyllidou et al. state that after poor response to an ovarian stimulation treatment due to limited ovarian reserve, the steroid synthesis and the follicle recruitment might be increased by giving DHEA [8-9]. This means the number of maturing preantral follicles and possibly also the number of mature follicles could be increased. In contrast, there are also authors who do not recommend androgen substitution with women with fertility problems for lack of evidence. Yeung et al., for example, published a small placebo controlled randomized study: 16 women with poor response were gaining DHEA. This did not affect the pregnancy rate [10]. Only limited data has been available so far to explain the benefit of an androgen treatment with women with fertility problems. For this reason, the American Endocrine Society does not recommend androgen substitution in its guidelines. A severe problem can be seen in the fact that any clear definition of the androgen deficiency syndrome in sterile women does not exist. 
All in all androgen substitution in women with unfulfilled wish for a child receiving fertility treatment might be a promising therapeutic approach with low risk-especially before initiating a therapy with multi-follicular stimulation. The challenges for the future will be to create more clinical evidence with the aid of prospective clinical trials and to define the syndrome of androgen deficiency in women with sterility with the possibility to find groups of patients who might benefit from a pre-therapeutic androgen supplementation.

\section{Acknowledgement}

None.

\section{Conflict of Interest}

The author reports no conflict of interest.

\section{References}

1. Sexty RE, Hamadneh J, Rösner S et al (2016) Cross-cultural comparison of fertility specific quality of life in German, Hungarian and Jordanian couples attending a fertility center. Healt Qual Life Outcomes 14: 27.

2. Berntsen S, Söderström-Anttila V, Wennerholm UB, Laivuori H, Loft A, et al. (2019) The health of children conceived by ART: 'the chicken or the egg?' Hum Reprod Update 25(2): 137-158.
3. Venturella R, Vaiarelli A, Cimadomo D, Pedri S, Lico D, et al. (2019) State of the art and emerging drug therapies for female infertility. Gynecol Endocrinol 29: 1-7.

4. Norman RJ, Dewailly D, Legro RS, Hickey TE (2007) Seminar: Polycystic Ovary syndrome. The Lancet 370: 685-697.

5. Hossein Rashidi B, Hormoz B, Shahrokh Theraninejad E, Shariat M, Mahdavi A (2009) Testosterone and dehydroepiandrosterone sulphate levels and IVF/ICSI results. Gynecol Endocrinol 25(3): 194-198.

6. Findeklee S, Sklavounos P, Stotz L, Sima RM, Iordache I, et al. (2019) Influence of androgen levels on conception probability in patients undergoing fertility treatment: a retrospective cohort study. Arch Gynecol Obstet 299(5): 1481-1485.

7. Dawood AS, El-Sharawy MA, Nada DW, El-Sheikh MF (2018) Premature ovarian failure of autoimmune etiology in 46XX patients: is there a hope? J Complement Integr Med 15(4).

8. Narkwichean A, MaaloufW, Campbell BK, Kannamannadiar Jayaprakasan (2013) Efficacy of dehydroepiandrosterone to improve ovarian response in women with diminished ovarian reserve: a meta-analysis. Reprod Biol Endocrinol 11: 44.

9. Triantafyllidou O, Sigalos G, Vlahos N (2017) Dehydroepiandrosterone (DHEA) supplementation and IVF outcome in poor responders. Hum Fertil (Camb) 20(2): 80-87.

10. Yeung TW, Chai J, Li RH, Lee VC, Ho PC (2014) A randomized, controlled, pilot trial on the effect of dehydroepiandrosterone on ovarian response markers, ovarian response, and in vitro fertilization outcomes in poor responders. Fertil Steril 102(1): 108-115. 